\title{
Revisiting graft-versus-host disease models of autoimmunity: new insights in immune regulatory processes
}

\author{
William J. Murphy
}

Transplantation Biology Laboratory, SAIC-Frederick, National Cancer Institute-Frederick Cancer Research and Development Center, Frederick, Maryland 21702, USA. Phone: (301) 846-5443; Fax: (301) 846-6641; E-mail: murphyw@ncifcrf.gov.

Graft-versus-host disease (GVHD) commonly occurs when immunocompetent donor cells attack a genetically disparate host. GVHD occurs primarily after allogeneic bone marrow transplantation (BMT) and remains a significant cause of morbidity, thus limiting the efficacy of this treatment. GVHD can exist as two distinct clinical entities: acute (affecting multiple solid organs) and chronic (presenting with solid organ pathology but also with autoimmune-like sequelae) $(1,2)$. New means to reduce the toxicity but retain the antitumor potential of BMT include donor lymphocyte infusions, nonmyeloablative conditioning, and the use of G-CSF to mobilize peripheral blood stem cells, all of which have led to significant reductions in the occurrence of acute GVHD (1). Unfortunately, with the decline of acute GVHD, chronic GVHD is emerging as a dominant complication in $\operatorname{BMT}(1,2)$.

Researchers examining GVHD have used numerous model systems, which has often resulted in confusion in extrapolating results from one model to another. GVHD models differ from one another with respect to three key features: the extent and type of conditioning or cytoreductive treatment of the recipient using total body irradiation or chemotherapy; the extent of genetic disparity between donor and host; and the purity, type, and number of donor cells transferred. These three variables have a dramatic impact on the type and extent of the resulting GVHD.

The parent-into-F1 model of GVHD One model that has been used involves the transfer of parental lymphocytes into nonconditioned F1 hybrid mice. Using this strain combination, the host $\mathrm{T}$ cells cannot actively resist the donor cells. When lymphocytes from
C57BL/6 mice are transferred into $(\mathrm{C} 57 \mathrm{BL} / 6 \times \mathrm{DBA} 2) \mathrm{F} 1$ recipients, an acute form of GVHD develops that affects solid organs and results in weight loss. Interestingly, when lymphocytes from the other parental strain, DBA2, are transferred into the same recipient animals, a chronic form of GVHD develops that resembles systemic lupus erythematosus with autoantibody production and glomerulonephritis (3). This chronic GVHD has been shown to be due to donor alloreactive $\mathrm{CD}^{+}{ }^{+} \mathrm{T}$-cell activation of host B cells (3). Concern over its relevance to current BMT protocols has dampened enthusiasm for this parent-into-F1 model of GVHD in favor of other GVHD models that use extensive conditioning and strain combinations that allow for immune reactivity on the part of both donor and host $\mathrm{T}$ cells. The advent of nonmyeloablative conditioning in transplants to reduce toxicity now alters that view. Such conditioning may employ minimal cytoreductive treatment (4) or may omit cytoreductive treatment entirely and rely solely on treatments that block the recipient's immune costimulatory molecules (5). This latter model more closely resembles the parent-intoF1 GVHD model, since neither involves cytoreductive conditioning, and, in both cases, recipient $\mathrm{T}$ cells are unresponsive to the donor.

The study by Shustov et al. presented in this issue of the JCI (6) emphasizes the relevance of the parent-into-F1 model, not only for GVHD and autoimmunity, but also potentially for understanding normal B-cell regulation. These authors demonstrate that one of the major mechanisms for cell killing, the perforin-dependent cytolysis pathway, plays a crucial role in the development of acute GVHD, in part through the suppression of chronic GVHD (6). Working in the C57BL/6into-F1 model, which ordinarily develops acute GVHD, they show that transfer of perforin-deficient donor lymphocytes instead results in chronic GVHD. Furthermore, they find that donor $\mathrm{CD}^{+} \mathrm{T}$ cells in this model are responsible for activating and expanding host $\mathrm{B}$ cells and that donor $\mathrm{CD}^{+}$ cells later suppress this expansion. The mice then develop acute GVHD pathology. However, when perforin-deficient donor $\mathrm{CD}^{+}$cells are transferred, they are unable to control the host B-cell expansion, and chronic GVHD pathology results (Figure 1). Interestingly, a cytokine shift also occurred in the mice receiving the perforin-deficient cells such that the Th2-type cytokines IL-10 and IL-4 predominated (6). It is still unclear whether B cells direct this Th2 shift by producing IL-10 or whether the mere expansion and presence of these activated $B$ cells dictates the nature of the cytokine response by the $\mathrm{T}$ cell. It also remains uncertain whether chronic GVHD in this system partially arises because perforin-deficient $\mathrm{T}$ cells are unable to induce optimal tissue destruction.

The perforin pathway of cell lysis Perforin has been previously demonstrated to play a pivotal role in cellmediated cytotoxicity, complementing the Fas/Fas ligand (FasL) pathway (7). Perforin, essentially a pore-forming protein capable of puncturing the cell membrane, is the primary mechanism underlying the lysis of target cells by natural killer cells (7). It has been also shown that granzyme-mediated destruction of tissue in models of GVHD is accentuated by perforin, presumably because perforin allows entry of granzymes into the target cells (8). 
Mutations in Fas or FasL, the other predominant pathway in cell-mediated cytotoxicity, have been associated with lymphoproliferative disorders, demonstrating their crucial role in lymphocyte homeostasis (9), but recent work has also drawn increasing attention to the role of perforin in regulating the immune response. Perforin knockout mice exhibit elevated immunoglobulin responses (10), and, when crossed onto genetic backgrounds that promote autoimmunity, perforin deficiency accelerates disease onset (11), thus establishing a role for perforin in regulating murine B-cell function. In addition, a human syndrome with T-cell and monocyte hyperplasia was recently shown to be due to a genetic defect in perforin (12). Nevertheless, it is still unclear how perforin, expressed in immunoregulatory cells, contributes to lymphocyte homeostasis.

The data of Shustov et al. (6) raise other interesting questions. Does perforin regulate B-cell expansion in other clinically relevant situations? For instance, the immune cell homeostasis is critical for the everyday suppression of herpes virus-infected B cells, notably Epstein Barr virus (EBV). Since $\mathrm{CD}^{+}$cells have been previously demonstrated to suppress EBVinduced B-cell lymphomas that can arise after immunosuppression or BMT (13), it is possible that perforin curbs B-cell expansion under these conditions. Hence, the ability to modulate this pathway would be of potential clinical use, either to inhibit B-cell expansion under pathologic conditions or to enhance B-cell recovery.

\section{Reconciling with other models of GVHD}

The extension of these findings to other models of GVHD also raises questions. Studies by Baker et al., using a model of acute GVHD that involved total body irradiation as a means of conditioning mice, demonstrated that not perforin but Fas/FasL interactions were the principal mediators responsible for inducing host myeloid and lymphoid hypoplasia (14). It is possible that conditioning alters the need for perforin by inducing other cytokines (TNF- $\alpha$ or IFN- $\gamma$ ), thereby inducing the Fas/FasL pathway of cell killing. The complicating effects of conditioning have been noted in studies of cytokine contributions to GVHD; depending on the extent of conditioning used, for example, cells from IFN- $\gamma$ knockout mice have markedly different effects on GVHD progression (15-17). Thus, although conditioning is not required for the initiation of GVHD, it must be taken into consideration before extrapolating the results and conclusions to other models.

The results of Shustov et al. (6) suggest that perforin should not be considered solely in the context of mediating cytotoxicity toward transformed and virally infected cells, as it may also play a significant role in immune

\section{Figure 1}

Potential role for perforin (pfp) in immune homeostasis. Mutations in perforin have been shown to be responsible for $\mathrm{CD} 8^{+} \mathrm{T}$-cell and macrophage hyperplasia, suggesting that immunoregulatory cells (possibly $\mathrm{CD} 8^{+} \mathrm{T}$ cells) use perforin for the suppression of these cells (blunt arrows). In a mouse model of GVHD, in which F1 hybrid animals receive lymphocytes from one of the parental strains (C57BL/6), donor $\mathrm{CD}^{+} \mathrm{T}$ cells promote host (shaded) B-cell expansion (pointed arrows). If unchecked, this expansion results in chronic GVHD. In this strain combination, donor $C D 8^{+} T$ cells mediate the elimination of the host $B$ cells (blunt arrow) and result in acute GVHD. EBV has been shown to infect B cells and can result in the development of an EBV lymphoma. It is suggested here that CD8 $8^{+} \mathrm{T}$ cells might use the perforin-dependent pathway of target cell killing to suppress the proliferation of such EBV-infected B cells.

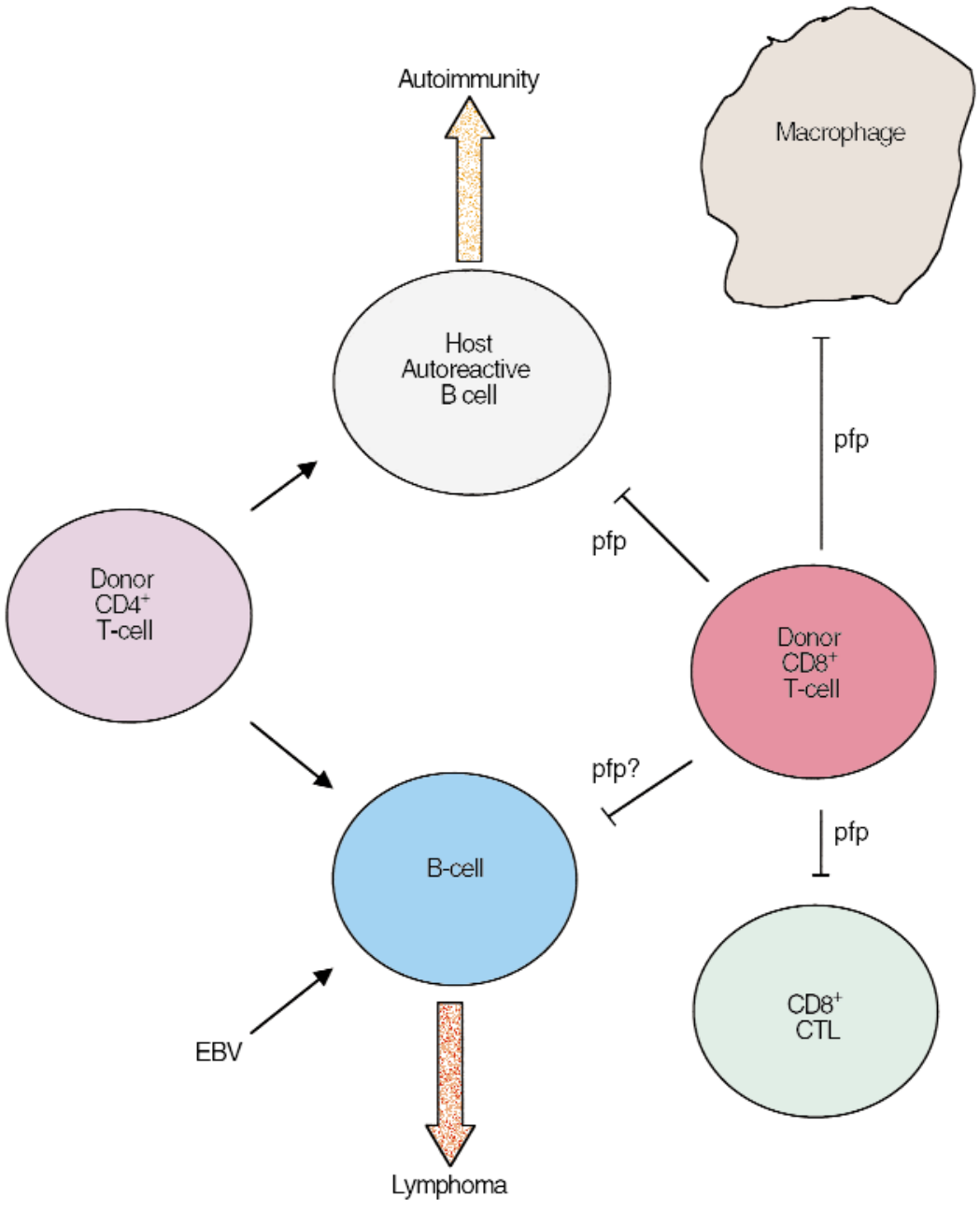


homeostasis. Further, with the emergence of nonmyeloablative therapies and the increasing incidence of chronic GVHD in BMT today, the parentinto-F1 hybrid model is also becoming increasingly relevant as a model for autoimmunity, GVHD, and immune homeostasis.

\section{Acknowledgments}

The content of this article does not necessarily reflect the views or policies of the Department of Health and Human Services, nor does mention of trade names, commercial products, or organizations imply endorsement by the US government.

This project has been funded in whole or in part with federal funds from the National Cancer Institute, NIH, under contract N01-CO-56000. I am particularly grateful to Michael Bennett, Bruce Blazar, Scott Durum, Dan Longo, Lisa Rogers, and Frank Ruscetti for helpful discussions and for reviewing the manuscript. I thank Lisbeth Welniak for help with the fig- ure. The excellent secretarial services by Laura Knott are also appreciated.

1. Murphy, W.J., and Blazar, B.R. 1999. New strategies for preventing graft-versus-host disease. Curr. Opin. Immunol. 11:509-515.

2. Parkman, R. 1993. Is chronic graft versus host disease an autoimmune disease? Curr. Opin. Immunol. 5:800-803.

3. Rus, V., et al. 1995. Kinetics of Th1 and Th2 cytokine production during the early course of acute and chronic murine graft-versus-host disease. Regulatory role of donor CD8+ T cells. $J$. Immunol. 55:2396-2406.

4. Sykes, M., et al. 1999. Mixed lymphohaemopoietic chimerism and graft-versus-lymphoma effects after non-myeloablative therapy and HLA-mismatched bone-marrow transplantation. Lancet. 353:1755-1759.

5. Kurtz, W., et al. 2000. Allogeneic bone marrow transplantation with co-stimulatory blockade induces macrochimerism and tolerance without cytoreductive host treatment. Nat. Med. 6:464-469.

6. Shustov, A., et al. 2000. Role of perforin in controlling B-cell hyperactivity and humoral autoimmunity. J. Clin. Invest. 106:R39-R47.

7. Pham, S.S., et al. 1998. How do cytotoxic lymphocytes kill their targets? Curr. Opin. Immunol. 10:581-587.

8. Graubert, T.A., et al. 1997. Perforin/granzymedependent and independent mechanisms are both important for the development of graft-versus-host disease after murine bone marrow transplantation. J. Clin. Invest. 100:904-911.
9. Zhang, H.G., et al. 2000. Antigen presenting cells expressing Fas ligand down-modulate chronic inflammatory disease in Fas ligand-deficient mice. J. Clin. Invest. 105:813-821.

10. Switzer, S.S., et al. 1998. Enhanced antibody and cytokine responses to influenza viral antigens in perforin-deficient mice. Cell Immunol. 87:13-18.

11. Peng, S.L., et al. 1998. Perforin protects against autoimmunity in lupus-prone mice. J. Immunol. 160:652-660.

12. Stepp, S.E., et al. 1999. Perforin gene defects in familial hemophagocytic lymphohistiocytosis. Science. 286:1957-1959.

13. O'Reilly, R.J., et al. 1997. Biology and adoptive cell therapy of Epstein-Barr virus-associated lymphoproliferative disorders in recipients of marrow allografts. Immunol. Rev. 157:195-216.

14. Baker, M.B., et al. 1997. Graft-versus-host-diseaseassociated lymphoid hypoplasia and B cell dysfunction is dependent upon donor T cell-mediated Fas-ligand function, but not perforin function. Proc. Natl. Acad. Sci. USA. 94:1366-1371.

15. Murphy, W.J., et al. 1998. Differential effects of the absence of interferon-gamma and IL-4 in acute graft-versus-host disease after allogeneic bone marrow transplantation in mice. J. Clin Invest. 102:1742-1748

16. Ellison, C.A., et al. 1998. Murine graft-versus-host disease in an F1-hybrid model using IFN-gamma gene knockout donors. J. Immunol. 161:631-640.

17. Shustov, A., et al. 1998. Differential expression of Fas and Fas ligand in acute and chronic graft-versus-host disease: up-regulation of Fas and Fas ligand requires CD8+ $\mathrm{T}$ cell activation and IFNgamma production. J. Immunol. 161:2848-2855. 